\title{
Clinical photoacoustic computed tomography of the human breast in vivo within a single breath hold
}

Li Lin, Peng Hu, Junhui Shi, Catherine M. Appleton, Konstantin Maslov, et al.

Li Lin, Peng Hu, Junhui Shi, Catherine M. Appleton, Konstantin Maslov, Lihong V. Wang, "Clinical photoacoustic computed tomography of the human breast in vivo within a single breath hold," Proc. SPIE 10494, Photons Plus Ultrasound: Imaging and Sensing 2018, 104942X (22 March 2018); doi: $10.1117 / 12.2290987$

SPIE. Event: SPIE BiOS, 2018, San Francisco, California, United States 


\title{
Clinical photoacoustic computed tomography of the human breast in vivo within a single breath hold
}

\author{
Li Lin, ${ }^{\mathrm{a}, \mathrm{b} \dagger}$ Peng Hu,${ }^{\mathrm{b} \dagger}$ Junhui Shi, ${ }^{\text {a }}$ Catherine M. Appleton, ${ }^{\mathrm{c}}$ Konstantin Maslov, ${ }^{\mathrm{a}}$
}

Lihong V. Wang ${ }^{\mathrm{a}, \mathrm{d} *}$

${ }^{a}$ Caltech Optical Imaging Laboratory, Andrew and Peggy Cherng Department of Medical Engineering, California Institute of Technology, 1200 East California Boulevard, Pasadena, CA 91125, USA.

${ }^{\mathrm{b}}$ Department of Biomedical Engineering, Washington University in St. Louis, One Brookings Dr., St. Louis, MO 63130, USA.

${ }^{c}$ Breast Imaging Section, Washington University School of Medicine in St. Louis, 510 South Kingshighway Blvd, St. Louis, MO 63108, USA.

${ }^{d}$ Caltech Optical Imaging Laboratory, Department of Electrical Engineering, California Institute of Technology, 1200 East California Boulevard, Pasadena, CA 91125, USA.

${ }^{\dagger}$ These authors contributed equally to this work.

*Corresponding author. Email: LVW@ caltech.edu

\begin{abstract}
We have developed a single-breath-hold photoacoustic computed tomography (SBH-PACT) system to detect tumors and reveal detailed angiographic information about human breasts. SBH-PACT provides high spatial and temporal resolutions with a deep in vivo penetration depth of over $4 \mathrm{~cm}$. A volumetric breast image can be acquired by scanning the breast within a single breath hold ( 15 sec). We imaged a healthy female volunteer and seven breast cancer patients. SBH-PACT clearly identified all tumors by revealing higher blood vessel densities and lower compliance associated with the tumors.
\end{abstract}

Keywords: Single breath hold, breast imaging, photoacoustic computed tomography, elastography, angiogenesis

Photons Plus Ultrasound: Imaging and Sensing 2018, edited by Alexander A. Oraevsky, Lihong V. Wang, Proc. of SPIE Vol. 10494, 104942X · (c) 2018 SPIE · CCC code: 1605-7422/18/\$18 · doi: 10.1117/12.2290987 


\section{INTRODUCTION}

Breast cancer is the second most common cause of cancer-related deaths in the U.S. [1]. While x-ray mammography is currently the gold standard for detecting breast cancer, it utilizes ionizing radiation and has low specificity [2]; it also has low sensitivity in women with dense breasts [3]. Breast MRI typically employs IV contrast agents that may cause kidney damage [4], and it requires protracted scans ( 45 min.) [5]. Ultrasonography can be an adjunct modality, but its reliability depends on the operator's experience $[6,7]$. To provide highly sensitive functional optical contrasts, diffuse optical tomography has been investigated. However, it suffers from poor spatial resolutions [8]. Overall, each modality has notable advantages and limitations.

Photoacoustic (PA) computed tomography (PACT) is a promising imaging modality that overcomes many of these limitations [9]. For breast imaging, PACT offers sufficiently deep nonionizing optical penetration [10] as well as high spatiotemporal resolutions. As the principal optical absorber in the near-infrared region, hemoglobin provides an endogenous contrast to reveal angiogenesis [11-13] and hypoxia [14, 15], which play important roles in tumor growth and metastasis [16].

Here, we report a single-breath-hold PACT (SBH-PACT) system that features a deep penetration depth (over $4 \mathrm{~cm}$ in vivo) with high spatial and temporal resolutions $(255-\mu \mathrm{m}$ in-plane resolution and a $10-\mathrm{Hz} 2 \mathrm{D}$ frame rate). Scanning the entire breast within a single breath hold $(\sim 15 \mathrm{sec})$ yields a volumetric breast image with detailed angiographic structures and negligible breathing-induced motion artifacts. In conjunction with anatomical imaging, SBH-PACT's high imaging speed allowed us to perform PA elastographic measurements, which disclosed stiffer tumor regions and provided another contrast to detect breast cancer. SBH-PACT also monitored blood flow-mediated deformations at the heartbeat frequency and mapped the arterial networks in breasts.

\section{RESULTS}

\subsection{Performance assessment on healthy breasts}

Each subject was positioned prone with one breast dependent through a large aperture in a bed. An agar pillow underneath the breast lightly pressed it against the chest wall (Fig. 1). Beneath the bed, a 1064-nm laser beam was expanded into a donut shape and directed upward to illuminate the breast. To provide 2D panoramic acoustic detection, we used a 512-element full-ring ultrasonic transducer 
array that detected PA waves around the breast. The transducer array was motorized by a linear stage to elevationally scan the breast. Four sets of 128-channel preamplifiers and acquisition systems were connected to the ultrasonic array. The data acquisition systems were set to record PA signals within $100 \mu$ s after each laser pulse excitation.

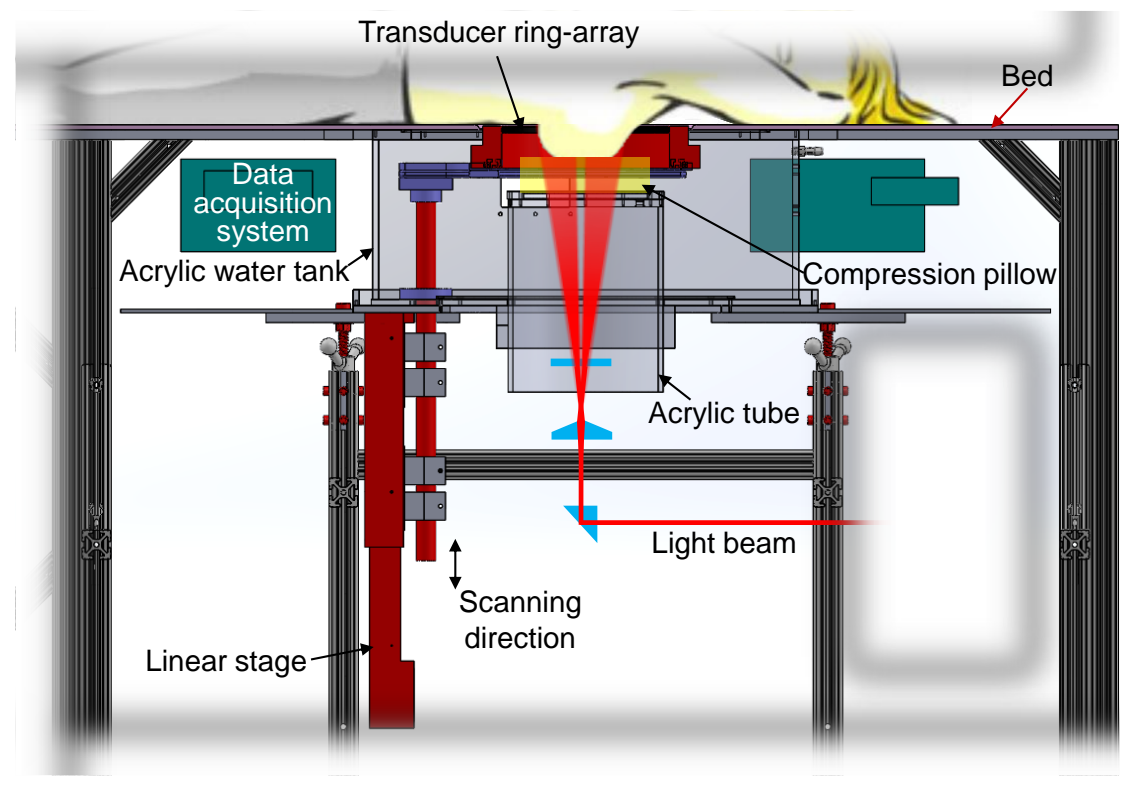

Figure 1. Perspective cut-away view of SBH-PACT.

Before imaging breast cancer patients, we first imaged a healthy female volunteer to optimize and assess the performance of the system. By scanning within a single breath hold of 15 seconds, SBH-PACT clearly revealed the detailed angiographic structures of the entire breast, visualizing the vasculature down to an apparent vascular diameter of $258 \mu \mathrm{m}$. By continuously monitoring arterial pulsatile deformations, SBH-PACT identified arteries and veins in a breast cross section.

\subsection{SBH-PACT of breast cancer anatomy, segmentation, and elastography}

Angiogenesis is the essential hallmark by which SBH-PACT identifies lesions. Enclosed by white dashed circles, a breast tumor is clearly visible in the depth-encoded image of Fig. 2. The tumor's position was well correlated with the results of x-ray mammograms (Fig. 2a) and ultrasound-guided biopsies. 


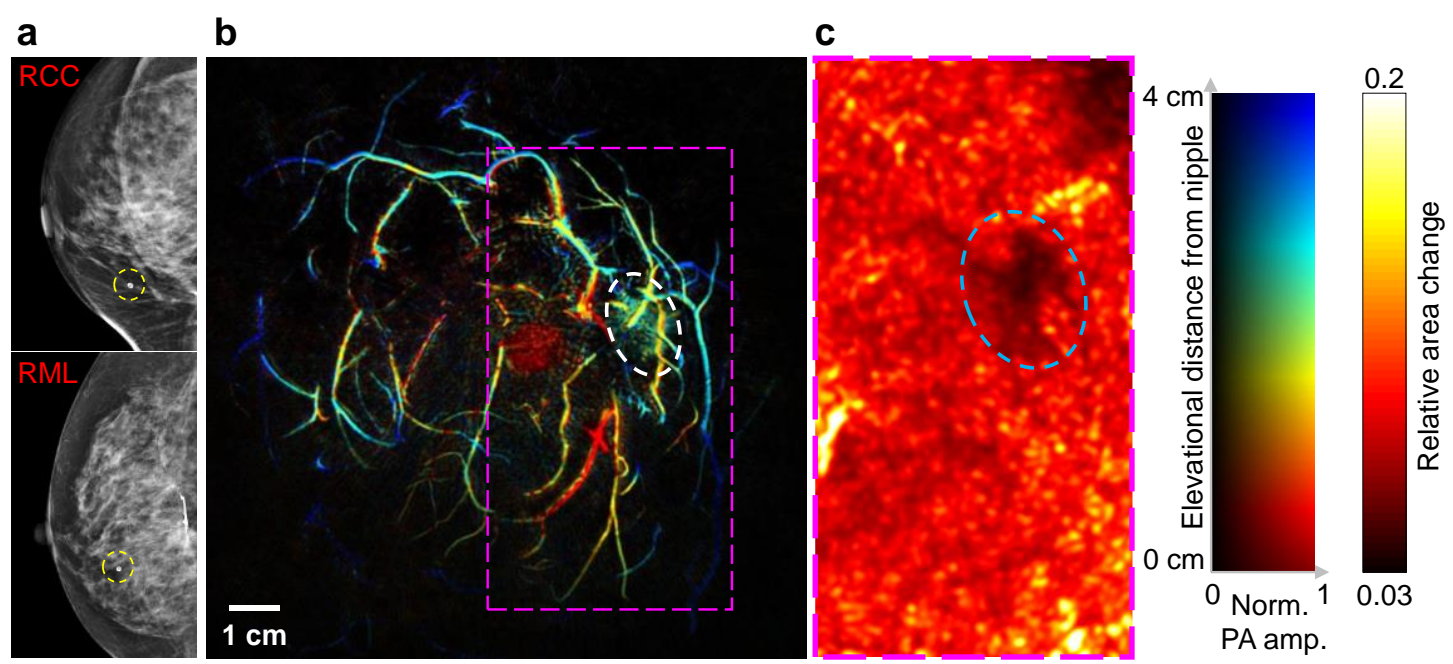

Figure 2. (a) X-ray mammograms of a 69-year-old female patient with an invasive ductal carcinoma (grade 2/3). RCC, right cranial-caudal; RML, right medio-lateral. (b) Depth-encoded angiogram of the same affected breast, acquired by SBH-PACT. (c) A map of the relative area change during breathing in the region outlined by the magenta dashed box in (b).

Other than directly observing angiogenesis, the difference in compliance between tumors and surrounding normal breast tissue was also revealed by SBH-PACT. Different from ultrasonic elastography, SBH-PACT-elastography evaluated area-quantificational grids between vessels. We first demonstrated this method on breast-mimicking phantoms and then applied it to image affected breasts. To assess minor deformations caused by breathing, SBH-PACT continuously quantified the relative area changes in a breast cross section. Because breast tumors are generally stiffer than normal breast tissue [17], the region with lower area changes indicated the breast tumor (Fig. 2c).

In assessing angiogenesis via angiograms, the only tumor we missed was located in a marginal region of a D cup breast, where we did not deposit enough light. However, SBH-PACT-elastography revealed this tumor by identifying the stiffer tumor region. Taking advantage of the short time requirement for the elastographic measurement ( $10 \mathrm{sec})$, SBH-PACT can improve the sensitivity of breast cancer detection by imaging both angiographic anatomy and tissue compliance within $\sim 30$ sec.

\subsection{Statistics}

We imaged a healthy female volunteer and seven breast cancer patients. Breast sizes ranged from B cup to DD cup, and skin pigmentations ranged from light to dark. By revealing higher blood vessel 
densities, SBH-PACT clearly identified eight of the nine biopsy-verified tumors. We developed a tumor demarcation algorithm by quantifying vessel density distributions of the breast. The average vessel density ratios between the tumors and the normal breast tissues were $3.4 \pm 0.99$. The elastography study began with Patient 4, and SBH-PACT elastography identified five tumors in the subsequent four patients. The average breath-induced area change in tumors was about two times lower than that of normal breast tissue.

\section{DISCUSSION}

We have developed an SBH-PACT system that achieved sensitive breast cancer detection with high spatiotemporal resolutions. Combining 1064-nm light illumination and 2D panoramic in-plane acoustic detection, SBH-PACT achieved up to $4 \mathrm{~cm}$ of imaging depth in vivo and a $255-\mu \mathrm{m}$ in-plane resolution. SBH-PACT could obtain an entire 2D cross-sectional breast image with a single laser pulse or obtain a volumetric 3D image of the entire breast by fast scanning within a single breathhold of 15 seconds.

The invention of this single-breath-hold breast imager provides a promising tool for future clinics. SBH-PACT has opened new doors for comprehensive breast cancer studies, including not only screening, but also diagnosis, definitive surgical treatment planning, and assessment of response to pre-operative systemic therapy. Compared to x-ray mammography, SBH-PACT utilizes non-ionizing radiation and has equally good sensitivity in detecting tumors in dense breasts. Using hemoglobin as the contrast, SBH-PACT can provide information similar to that of contrast-enhanced MRI, yet with finer spatial resolution, higher imaging speed, and endogenous contrast. These advantages give SBH-PACT the potential to monitor breast cancer responses to neoadjuvant chemotherapy.

\section{ACKNOWLEDGMENTS}

The authors would like to thank $\mathrm{Xi} \mathrm{Li}$ and Ruth Holdener for useful discussions and patient recruitment. We are grateful to James Linders for technical support. We also thank Prof. James Ballard and Amy Leong for close reading of the paper. This work was sponsored by the United States National Institutes of Health (NIH) grants DP1 EB016986 (NIH Director's Pioneer Award), R01 CA186567 (NIH Director's Transformative Research Award), and R01 EB016963. 


\section{REFERENCES}

[1] Siegel, R.L., K.D. Miller, and A. Jemal, Cancer Statistics, 2017. CA Cancer J Clin, 2017. 67(1): p. 7-30.

[2] Popli, M.B., R. Teotia, M. Narang, et al., Breast Positioning during Mammography: Mistakes to be Avoided. Breast Cancer (Auckl), 2014. 8: p. 119-24.

[3] Pinsky, R.W. and M.A. Helvie, Mammographic breast density: effect on imaging and breast cancer risk. J Natl Compr Canc Netw, 2010. 8(10): p. 1157-64; quiz 1165.

[4] Perazella, M.A., Gadolinium-contrast toxicity in patients with kidney disease: nephrotoxicity and nephrogenic systemic fibrosis. Curr Drug Saf, 2008. 3(1): p. 67-75.

[5] Morris, E.A., Rethinking breast cancer screening: ultra FAST breast magnetic resonance imaging. 2014, American Society of Clinical Oncology.

[6] Lehman, C.D., C. Isaacs, M.D. Schnall, et al., Cancer yield of mammography, MR, and US in high-risk women: prospective multi-institution breast cancer screening study. Radiology, 2007. 244(2): p. 381-8.

[7] Corsetti, V., N. Houssami, A. Ferrari, et al., Breast screening with ultrasound in women with mammography-negative dense breasts: evidence on incremental cancer detection and false positives, and associated cost. Eur J Cancer, 2008. 44(4): p. 539-44.

[8] Leff, D.R., O.J. Warren, L.C. Enfield, et al., Diffuse optical imaging of the healthy and diseased breast: a systematic review. Breast Cancer Res Treat, 2008. 108(1): p. 9-22.

[9] Wang, L.V., Multiscale photoacoustic microscopy and computed tomography. Nat Photonics, 2009. 3(9): p. 503-509.

[10] Garcia-Uribe, A., Erpelding, T. N., Krumholz, A., et al., Dual-modality photoacoustic and ultrasound imaging system for noninvasive sentinel lymph node detection in patients with breast cancer. Sci. Rep., 2015. 515748.

[11] Reynolds, A.R., I.R. Hart, A.R. Watson, et al., Stimulation of tumor growth and angiogenesis by low concentrations of RGD-mimetic integrin inhibitors. Nat Med, 2009. 15(4): p. 392-400.

[12] Schneider, B.P. and K.D. Miller, Angiogenesis of breast cancer. J Clin Oncol, 2005. 23(8): p. 1782-90.

[13] Weidner, N., J.P. Semple, W.R. Welch, et al., Tumor angiogenesis and metastasis--correlation in invasive breast carcinoma. N Engl J Med, 1991. 324(1): p. 1-8.

[14] Gilkes, D.M. and G.L. Semenza, Role of hypoxia-inducible factors in breast cancer metastasis. Future Oncol, 2013. 9(11): p. 1623-36.

[15] Vaupel, P., A. Mayer, S. Briest, et al., Hypoxia in breast cancer: role of blood flow, oxygen diffusion distances, and anemia in the development of oxygen depletion. Adv Exp Med Biol, 2005. 566: p. 333-42.

[16] Folkman, J., Role of angiogenesis in tumor growth and metastasis. Semin Oncol, 2002. 29 (6 Suppl 16): p. 15-8. 
[17] Wellman, P. S., Howe, R. D., Dalton, E. et al., Breast tissue stiffness in comperssion is correlated to histological diagnosis. Technical Report. Harvard BioRobotics Laboratory, 1999. p. $1-15$. 